\title{
Trends of using of the cone beam computed tomography imagery in oral and maxillofacial medical dentistry in the future in Albania
}

\begin{abstract}
Generator system of the Cone Beam Computed Tomography (CBCT) is a new machine, which rapidly is using for every speciality in dental practices in Albania. The different ways of viewing CBCT data sets and guides clinicians in identifying familiar and unfamiliar anatomical landmarks for three planes of section (three dimensional 3D), axial, sagittal, and coronal. Conventional intraoral radiography and panoramic radiography offer only two dimensional (2D) view of the region of interest in buccal-lingual perspectives. For a long time clinicians have experimented and expressed the need for three dimensional (3D) images view of the teeth, jaws and surrounding structures, in order to improve diagnosis and treatment planning. At the 2D system, superimpositions are in limited value, for detecting subtle anatomical and pathological structures and for that reason using of 3D images has become increasingly prominent in dental radiology. However, up to 2000 in Albania, there was a lack of technique for the $3 \mathrm{D}$ anatomy of teeth and jaws to be captured in a high quality yet simple, affordable manner with minimal dosage. The solution came from using of new technique of CBCT designated for high resolution imaging for examination or treatment of teeth, jaws and hard tissues. This is a big advantage today in dental diagnosis, because has proven affective for applications in all fields of dentistry abroad.
\end{abstract}

Keywords: Cone beam computed tomography, 3D (three dimensional) planes axialsagittal and coronal, dental radiology, radiation exposure dose
Volume 9 Issue 4 - 2018

\author{
Emil Qafmolla,' Luan Qafmolla, ${ }^{2}$ Rushdie \\ Qafmolla $^{3}$ \\ 'MSc Private Dental Clinic, Albania \\ ${ }^{2}$ Private Consultant for Radiation Protection, Albania \\ ${ }^{3}$ ALDENT University, Albania
}

Correspondence: Luan Qafmolla, Private Consultant for Radiation Protection, Tirana, Albania,

Email I_qafmolla@hotmail.com

Received: September 06, 2017 | Published: August 22, 2018

\section{Introduction}

Medical technology has been improving at an incredible rate for the past several decades; leading to leaps and bounds in the quality of care for people abroad and especially in Albania and this is significant true for the dental industry. One of the most important and exciting developments in dental diagnostic technology in the past decade has been the advent of $2 \mathrm{D}$ and 3D dental imaging, respectively by intraoral, panoramic and cone beam computed tomography machines. Since the introduction of computed tomography (CT), digital 3D imaging has become increasingly prominent in dental radiology. 3D dentistry, at its most basic, involves developing a digital, 3D image of the mouth and skull as well as those is designated for high resolution imaging of hard tissues. However, most people likely aren't even aware of: what 3D dental technology is and that's how new it is; what is $3 \mathrm{D}$ dental imaging and why is $3 \mathrm{D}$ dentistry better for imaging radiography of the patients? Answer is: $3 D$ dental imaging is well on its way to becoming new standard of dental care.

This incredible technological advancement allows dentists to see the mouth of patients in a way, never previously possible with X-ray or medical CT scan technology. 3D dental imaging allows them to see the teeth, skull and hard tissues, to diagnose any problems and provide more effective treatment of patients. ${ }^{1,2} 3 \mathrm{D}$ dental images are most often used for diagnosis and treatment planning. Being able to see the mouth in three dimensions allows the specialist dentist to better and more effectively formulate an approach to treat dental conditions. Cone beam CT has particular operational characteristics that make high resolution imaging possibility.

Firstly, an X-ray tube and a 2D image detector are mounted opposite each other and perform one rotation $180^{\circ}-360^{\circ}$ around region of interest.

Secondly, the resulting primary data, up to 600 projections are converted into slice data using the filtered back projection.

Thirdly, the reconstructed slice data can be viewed in user - defined planes according to the requirements of the dentist. CBCT technology and procedures can be useful for all areas of dentistry dealing with mouth, skull and hard tissues diagnostic, but is of limited value for the demonstration of different soft tissues. The main applications of CBCT based in clinical practices in dentistry and in research experiences of dental institutions are as following

Dent-maxilla-facial surgery where impacted or displaced teeth, apical periodontitis and other chronic inflammation of the jaw, cysts of the jaw, pre-and postoperative imaging for noting important anatomical landmarks and structures, implant treatment planning and recall, cleft-palate malformation cases, trauma cases (bone and teeth fractures) and examination of the maxillary sinus are some important areas of using of CBCT applications. ${ }^{3}$

Periodontology is another important area, where this dental technology may to investigate strictly marginal bone contouring, 3D imaging of deep pockets and furcations in bone. ${ }^{4}$

Endodontic investigated the configuration of the root canals, root canal measurements, additional canals and anatomical variations. ${ }^{1,2,5}$

Orthodontics is also an important area, where the CBCT technology may to be applied with high interest at the configuration of roots, anatomical situation, relation of teeth between periodontal ligament and resorption. 


\section{Terms and procedures of using of the CBCT dental technology}

\section{Terms of using a CBCT technology (machine) in medical applications}

Today, cone-beam computed tomography is used in the fields of dentistry, radiotherapy and mammography, among others, and it's used as a safer and more effective alternative to medical CT scans. The number of dental practices, dental and otherwise; using CBCT units in Europe is experiencing a steady rise as more people discover the incredible benefits of CBCT technology. This growth is only hindered by the cost of implementing the system. Along with this steady growth of implementation, professionals around the world are in the process of developing new standards and applications for this technology. Currently, worldwide dentistry organizations are developing standards of practice for using CBCT units safely, while others are theorizing potential new uses for the technology in science and medical research. ${ }^{6}$ The cone-beam computed tomography CBCT machine, is widely regarded as the new pinnacle of 3D imaging technology, and it represents the official shift from 2D imaging to $3 \mathrm{D}$ imaging. This machine uses a cone-shaped X-ray beam to take pictures of the skull and teeth, and then it uses those pictures, along with robust digital processing software, to reconstruct a 3D image. Provided the settings are correct and the patient does not move during the image-collecting process, this image is extremely accurate, with few, if any, distortions in Figure 1.
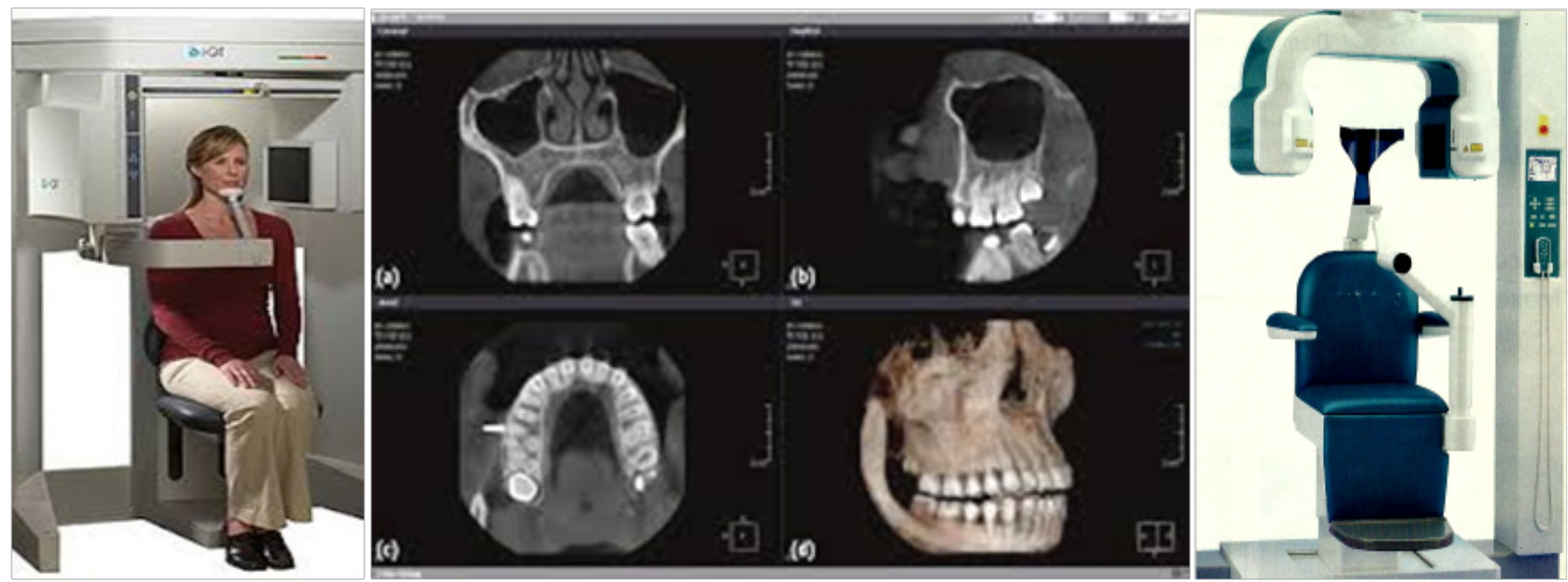

Figure I Cone beam computed tomography device \& teeth, jaws, skull skeletal views.

Function and operational aspects of CBCT machine in medical applications

This unique process results in an extremely useful digital model, which can be referenced for diagnostic and treatment purposes. It also sets it apart from previous methods as a more advanced option and more detailed description of the process for the using of this kind of machine is as follows

Situating the patient depending on the type of cone beam imaging system used, the subject may be positioned in a supine, standing or sitting position, with the head or area of interest placed at the center of the CBCT system. Of these positions, sitting is most common, as it is most comfortable and sustainable for the patient and is more accommodating for disabled patients. Upon situating the patient in place, the patient's head will be stabilized with a restraint mechanism, such as a chin rest, to minimize movement during the scanning process.

Acquiring the scan the X-ray source rotates around the head, while a reciprocating area detector moves opposite, both rotating around a fixed fulcrum oriented directly over the patient's head. The frame rate, speed of rotation, field of view and completeness of the trajectory arc are all set to get the image desired by the dental practitioner. During this process, the machine emits radiation in short bursts, meaning the exposure time of the patient is only a fraction of the total scan time. This scan takes about ten seconds on average.
Detecting the image the detector picks up the radiation emitted from the X-ray and collects it as digital data. This data is then transmitted to and collected at a dedicated computer.

Reconstructing the image once the projected images have been acquired and collected, the 100 to 600 images are taken and used to construct a model of the skull. This is done with a software that uses reconstruction algorithms to build up a three dimensional model of the skull, teeth or other area of interest. This can take several minutes to complete, depending on the quality of the software and computer hardware.

Displaying the image, $\mathrm{CBCT}$ technology provides a complete digital model at the end of the process. The software also provides the dental clinician with a relatively large choice of display formats, allowing for 2D, 3D and panoramic views of the mouth and head, along with other viewing options to help focus in on areas of interest. ${ }^{6,7}$

\section{Comparisons and advantages of 3D in dentistry with medical computing tomography (CT) scan}

The unique way $3 \mathrm{D}$ dentistry works means it offers numerous features found nowhere else in dental technology. This technology has a huge number of advantages over technologies like traditional $\mathrm{X}$-ray and medical CT scans. The main advantages of the CBCT in comparison with spiral computing tomography are the higher resolution, the significantly lower radiation burden and that artifacts, 
caused by metallic structures are much less disturbing.

The cone beam computing tomography obtains a spatial resolution up to 4 line pairs $/ \mathrm{mm}$, while the best medical CT machines only 2 line pairs $/ \mathrm{mm}$. The high resolutions are necessary to display subtle structures like root canals or periodontal ligament.

Short scan time because the CBCT scans involved in 3D dentistry can acquire all scan images in a single rotation, scan time isn't much different from a normal scan. This reduces the chance of image defects due to the natural movement of the patient. It also minimizes time spent on the scan.

Image accuracy $\&$ image details $3 \mathrm{D}$ CBCT imaging has an incredible resolution, allowing for highly accurate imaging and measuring. This means these images can be used to pinpoint the exact location for a dental procedure. With a single scan of a CBCT machine, dentists can see much more than a regular X-ray. They can see pathology, infections, nerves, musculature and so much more. This helps dentists see and properly treat dental-caused sinus issues and plan for root canals, implants and extractions. The possibilities are endless with $3 D C B C T$ scans.

Bone quality assessment 3D CBCT scans can also be used to assess bone quality, which is an important part of evaluating the presence of sufficient bone for implant placement. It is also helpful in determining the size and location of lesions and breaks.

User-friendly 3D dental equipment is very easy to learn, and it's designed for a trained dentist or dental technician to learn and work with easily. ${ }^{4,6-8}$

\section{Radiation dose consideration and beam limitation during CBCT procedures}

The size of the primary X-ray beam in 3D CBCT scanners limits the radiation to only the area of interest, reducing the patient's exposure to radiation as much as possible. From the radiation protection point of view, CBCT machine is by far superior to spiral CT regardless of the CT generation. Of course, the radiation dose is dependent on the field of view (FOV). ${ }^{9,10}$ For instance if used the 3D Accuitoma with a cylindrical field of view of $30 \mathrm{~mm}$ in height and $40 \mathrm{~mm}$ in diameter, the radiation burden is low as two additional days of natural background in Albania. We have experiment the measurement of dose rate at patients as below

\section{Material \& method}

TLD chips were used to record the distribution of the absorbed radiation dose at selected locations in the head and neck region of a small adult skull and tissue-equivalent phantom (Figure 2). The 24 sites at head and neck RANDOM phantom were measured in our study (In National Personnel Dosimetry Laboratory, Prague, Czech Republic), which are located and reflect critical organs known to be sensitive to radiation, along with the eyes and the pituitary gland, which are sites of traditional and topical interest, respectively, to dental imaging. An unexposed dosimeter was also included for environmental calibration of each technique run. Pre-calibrated $3 \mathrm{~mm} \times 3 \mathrm{~mm} \times 1 \mathrm{~mm}$, Thermo Luminescent Dosimeter (TLD-100) lithium fluoride chips were supplied and analyzed by Harshaw Reader HR-6000 device. In this device was calibrated each dosimeter by exposing it to a known quantity of radiation from a ${ }^{137} \mathrm{Cs}$ standard radioactive source. Dosimeters were analyzed using an automatic hot gas reader and the raw data were recorded. Individual TLD chip sensitivity was obtained and applied as a correction factor to subsequent exposure and reading of each TLD. The standard deviation of calibrated readings from the supplied TLD 100 chips is stated to be less than $\pm 5 \%$. Thermo-luminescent dosimeters (TLDs-100) were placed at 24 sites throughout the layers of the head and neck of a tissue-equivalent human skull of phantom. Depending on availability, the 12" (inch) field of view (FOV) and smaller FOV scanning modes were used with similar phantom positioning geometry for CBCT unit. Radiation weighted doses to individual organs were summed using $1990\left(E_{1990}\right)$ and proposed $2005\left(E_{2005 \text { draft }}\right)$ ICRP tissue weighting factors to calculate two measures of whole-body effective dose. Dose as a multiple of a representative panoramic radiography dose was also calculated.

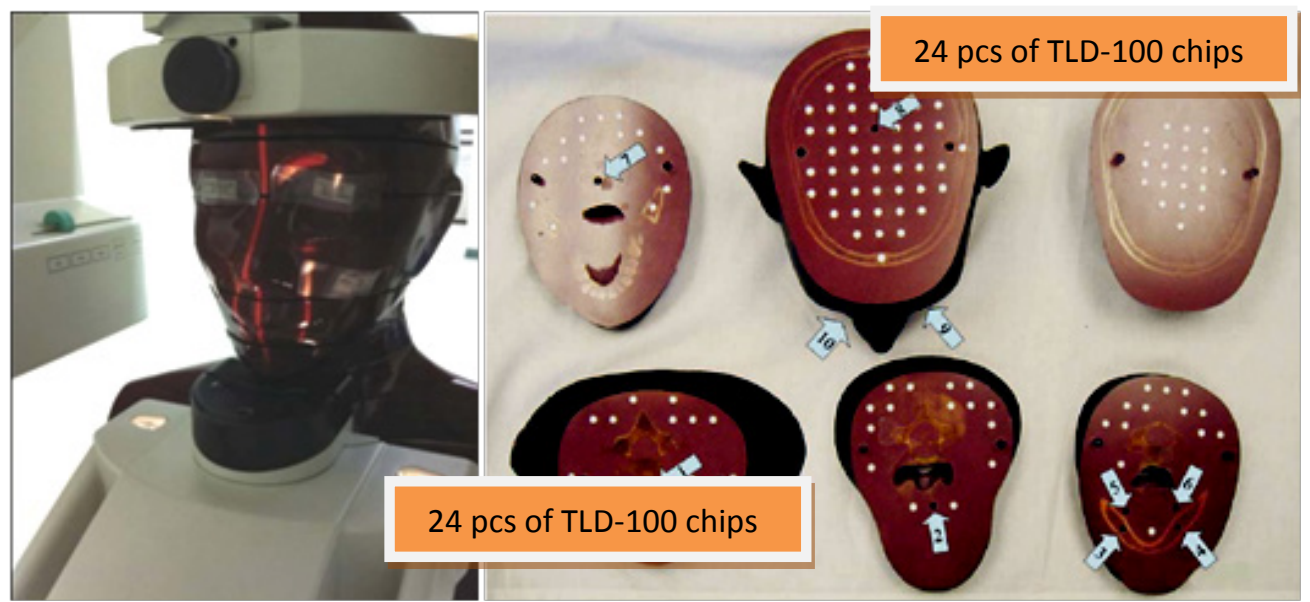

Figure 2 Phantom for evaluation of radiation dose during CBCT examinations using TLD-I00 chips.

\section{Results}

For repeated runs dosimetry was generally reproducible within $2.5 \%$. Calculated doses in miliSivert $(\mathrm{mSv})$ were performed for both NewTom $3 G$ and $i$-CAT machines. These are 3 to 30 (maximal values) times greater than comparable panoramic examination doses $((\cong 3 \mathrm{mSv}, \cong 9 \mathrm{mSv})$. Reductions in dose were seen with reduction in field size and parameters of machines to $\mathrm{mA}$ and $\mathrm{kV}$ technique factors. 
Radiographic techniques have to be evaluated concerning radiation dose, which requires well-defined and easy-to-use CT dose index (CTDI - Computing Tomography Dose Index), prevailing method for CT units, and (DAP-dose-area product) were evaluated for calculating effective dose (E) for both units. An asymmetric dose distribution was revealed when a clinical situation was simulated. Based on DAP values from 60 patients examinations effective dose was estimated for three diagnostic tasks: implant planning in posterior mandible and examinations of impacted lower third molars and retained upper cuspids. It varied between $\cong 8-60 \mu \mathrm{Sv}$ (microsivert). ${ }^{9,10}$ Usually the radiation dose should be evaluated together with image quality and if will be adapted the exposure parameters to diagnostic task can give substantial dose reduction. When was used the Imaging Science International (i-CAT device Imaging Science International) with field of view of $130 \mathrm{~mm}$ in high and $130 \mathrm{~mm}$ in diameter, the radiation dose is approximately $1 / 4$ of comparable CT scan. Lowered dosage of the cone beam computing tomography is of great benefit to younger patients with cleft palate or orthodontic malformations, who require regular X-ray imaging provided by $3 \mathrm{D}$ machine. Also, using of such devices, especially in dental application and generally in medical treatment, are totally in accordance with ALARA (as low as radiation $\boldsymbol{a}$ chievable) principle of radiation protection, for justifying of medical procedures to the patients. ${ }^{9-11}$

Occupational exposure from $\mathrm{CBCT}$ should not be an issue when such equipment is correctly installed. The CBCT supplier and the Radiation Protection Advisor (RPA) should usually collaborate to design the facility in which the CBCT scanner is to be housed with appropriate shielding to protect staff during exposure. CBCT units are capable of greater power and X-ray scatter than conventional dental $\mathrm{X}$-ray units and would normally require a dedicated room where the operator may stand outside or behind a suitable screen of brick or lead. During a conventional dental intra-oral X-ray exposure, many practitioners do not use shielding but stand a safe distance of over $1.5 \mathrm{~m}$ behind or to the side of the machine. Applying this principle, an operator would need to stand at least $8 \mathrm{~m}$ from a CBCT machine.

The average exposure to a patient during a $\mathrm{CT}$ examination is commonly estimated and presented as the Computed Tomography Dose Index (CTDI). All modern CT devices should display this figure, allowing for the estimation of the effect of different scan protocols on dose. However, this method of dose estimation is not available with all $\mathrm{CBCT}$ apparatus. An alternative approach for $\mathrm{CBCT}$ is to use the 'dose area product' (DAP), which estimates the exposure to the patient by directly measuring the incident $\mathrm{X}$-ray beam.

\section{Conclusion}

i. In summary, we have to become aware of the myriad possibilities that cone beam computing tomography offers dental x-ray diagnostics as above mentioned for diagnostic and treatment procedures.

ii. Also, in the near future, I am certain and believe that soon in Albania private dental clinics will be in use more and more cone beam computing tomography machines, in order that doctors and technicians to be more effectively in diagnostics and treatment procedures to the patients. iii. CBCT technology is increasingly accessible in dental practice. It hugely expands diagnostic and treatment possibilities for patients. However, CBCT should only be used after careful consideration, where conventional two-dimensional imaging techniques are not sufficient or where access to the technological processes such as guided surgery will improve patient management.

iv. When selecting the best CBCT examination for an individual, it is important to minimize X-ray dose, while striving for an image that enables appropriate diagnosis and management. This requires an understanding of the concepts behind $\mathrm{CBCT}$ and related technologies, making appropriate training essential for every member of the dental team.

\section{Acknowledgements}

None.

\section{Conflict of interest}

Author declares there is no conflict of interest.

\section{References}

1. Gröndahl HG, Huumonen S. Radiographic manifestations of periapical inflammatory lesions. Endodontic Top. 2004;8:55-67.

2. Whaites E. Periapical radiography in essentials of dental radiology and radiography, $4^{\text {th }}$ edition. Chapter 10. Churchill Livingston Elsevier. 2007.

3. Cohenca N, Simon JH, Roges R. Clinical indications for digital imaging in dent-alveolar trauma. Part 1: traumatic injuries. Dental Traumatic. 2007;23:95-104

4. Abu-Saleh, Tareq Heo, Young-Ku. Cone Beam CT in Dental Practice: A Surgical Perspective. Smile Dental Journal. 2014;9(3):10-13.

5. Patel S, Dawood A, Whaites E. New dimensions in endodontic imaging: part 1. Conventional and alternative radiographic systems. International Endodontic Journal. 2009;42:447-462.

6. Arai Y, Tammisalo E, Iwai K, et al. Development of a compact computed tomography apparatus for dental use. Dento-maxillofacial Radiology. 1999;28:245-248.

7. Hashimoto K, Kawashima S, Araki M, et al. Comparison of image performance between cone-beam computed tomography for dental use and four-row multi-detector helical CT. Journal of Oral Science. 2006;48:27-34

8. Ludlow JB, Davies-Ludlow LE, Brooks SL, et al. Dosimetry of 3 CBCT devices for oral and maxillofacial radiology. Dentomaxillofac Radiol. 2006;35:219-226.

9. Paloma JM, Hans MG, Rao PS. Influence of CBCT exposure conditions on radiation dose. Oral Surg Oral Med Oral Patho Oral Radiol Endod. 2008;105(6):773-782.

10. Loubele M, Bogaerts R, Van Dijck, et al. Comparison between effective radiation dose of $\mathrm{CBCT}$ and MSCT scanners for dent maxilla facial applications. Europe Journal Radiology. 2008;71(3):461-8.

11. Brand JW, Gibbs SJ, Edwards M. Radiation Protection in Dentistry. NCRP. 2003. 